G.O. Hofmann

Berufsgenossenschaftliche Unfallklinik Murnau

\title{
Retrograde Marknagelung bei proximalen Unterschenkelfrakturen
}

\section{Indikation}

Die Marknagelung von Tibiafrakturen gilt als eine weithin anerkannte Methode der Osteosynthese. Bislang konnten Marknägel für Frakturen und Osteotomien im kranialen Fünftel der Tibia aber nicht oder nur unter schwierigen Umständen zum Einsatz kommen, weil keine retrograde Marknagelungsimplantationstechnik zur Verfügung stand. Dagegen ist die retrograde Marknagelungstechnik bei der osteosynthetischen Versorgung von Frakturen an Femur und Humerus seit langem bereits klinische Routine.

Nach anatomischen und biomechanischen Voruntersuchungen wurde die retrograde Marknageltechnik für die Tibia entwickelt und anschließend in einer klinisch kontrollierten Studie zum Ersteinsatz geführt. Dabei zeigte sich, dass die retrograde Tibiamarknagelung bei ganz proximalen Tibiafrakturen und Osteotomien als ein wenig invasives, alternatives Verfahren zur Plattenosteosynthese angesehen werden kann.

\section{Schlüsselwörter}

Proximale Tibia · Retrograde Marknagelungstechnik · Tibiafraktur · Osteosynthese
Retrograde Marknagelungstechniken sind bei der osteosynthetischen Versorgung von Frakturen an Femur und $\mathrm{Hu}$ merus derzeit bereits klinische Routine $[1,2,3]$. Obgleich die Marknagelung von Tibiafrakturen in antegrader Richtung als eine weithin anerkannte Methode der Osteosynthese gilt, konnten Marknägel für Frakturen und Osteotomien im kranialsten Fünftel der Tibia bislang nicht oder nur unter schwierigen Umständen zum Einsatz kommen, weil keine retrograde Marknagelimplantationstechnik zur Verfügung stand.

Dabei ist der Gedanke der retrograden Tibiamarknagelung nicht neu. Küntscher u. Maatz [4] haben in ihrem Buch „Technik der Marknagelung“ bereits 1945 dieses Verfahren vorgestellt. In den Jahren 1999 und 2001 veröffentlichte unsere Arbeitsgruppe die Ergebnisse anatomisch-biomechanischer Untersuchungen zur retrograden Marknagelungstechnik an der Tibia [5] und stellte erste klinische Ergebnisse vor [6]. Den Anstoß zu dieser Entwicklung hatte unser allogenes Kniegelenkstransplantationsprojekt gegeben $[7,8]$. Für die Osteosynthese der sehr proximalen tibialen Osteotomie der allogenen Transplantate war, sollte eine intramedulläre Osteosynthesetechnik zur Anwendung kommen, das retrograde Einbringen und Vorschieben eines Marknagels in die proximale Tibia zwingend erforderlich.
Bald schon zeigten sich jedoch weitere mögliche Indikationen für eine retrograde Tibiamarknagelung:

- Frakturen im proximalen Tibiafünftel,

- Korrekturosteotomien im proximalen Tibiafünftel,

- Segmenttransport im proximalen Tibiadrittel (Marknagel in Kombination mit Seilzug) zur knöchernen Defektrekonstruktion,

- Unterschenkelfraktur bei vorbestehender Kniegelenksarthrodese.

\section{Operationstechnik}

Unsere anatomischen und biomechanischen Voruntersuchungen hatten gezeigt, dass der ideale Eintrittspunkt für eine retrograde Tibiamarknagelung in der Sagittalebene direkt oberhalb des oberen Sprunggelenkes liegt [5]. Der Patient liegt auf dem Rücken mit freibeweglich abgedecktem Bein. Als Zugang dient eine 3-5 cm lange Längsinzision an der Ventralseite der distalen Tibia in Höhe des oberen Sprunggelenkes. Subkutangewebe, Faszie und die Retinakula der Extensoren werden gespalten. Der M. extensor

(C) Springer-Verlag 2003

Prof.Dr.Dr. rer. nat. Gunther 0 . Hofmann Abteilung für septische und Wiederherstellungschirurgie, Berufsgenossenschaftliche Unfallklinik Murnau/Staffelsee, Prof.-Küntscher-Straße 8, 82418 Murnau, E-Mail: gunther.hofmann@bgu-murnau.de 
Trauma Berufskrankh 2003 - 5 [Suppl 2]: S235-S239 DOI 10.1007/s10039-002-0650-7

\section{G. O. Hofmann}

\section{Retrograde nailing in high tibial fractures}

\begin{abstract}
Intramedullary nailing of tibial fractures is a widely accepted method of osteosynthesis. Up to now intramedullary nails could not be employed for fractures and osteotomies in the most cranial fifth of the tibia, because no retrograde implantation technique was available. This situation is very different to femur and humerus, where retrograde implantation of intramedullary nails is clinical routine in osteosynthesis since a few years. Following anatomical and biomechanical studies we developed an operation and implantation technique for retrograde tibial nailing. This is the report of some clinical studies on the topic. The first results support retrograde tibial nailing as an advantageous and less invasive alternative to plate-osteosynthesis in some cases of very proximal tibial fractures and very high osteotomies.
\end{abstract}

\section{Keywords}

Retrograde tibial nailing - Osteosynthesis . Tibia fractures · Proximal tibia

\section{Unterschenkel- und obere Sprunggelenksfrakturen}

hallucis longus wird nach medial abgehalten. Zwischen diesem Muskel und dem M. extensor digitorum longus werden A. und V. tibialis anterior und der $\mathrm{N}$. peronaeus profundus präpariert und nach lateral abgehalten. Die Kortikalis der distalen Tibia wird subperiostal freigelegt, ohne dass dabei die Gelenkkapsel des oberen Sprunggelenkes eröffnet werden muss.

Unmittelbar oberhalb der Gelenkkapsel wird die Kortikalis der Facies ventralis der distalen Tibia mit dem Pfrim eröffnet. Ein Führungsdraht mit leicht gebogener Spitze wird in den Markkanal eingeführt und über die Fraktur bzw. Osteotomie in das proximale Fragment der Tibia direkt unter die Eminentia intercondylaris vorgeschoben. Unter dem Einsatz von flexiblen Bohrwellen wird der Markkanal der Tibia retrograd präpariert. Wir starten mit einem Bohrwellendurchmesser von $8 \mathrm{~mm}$ und erhöhen diesen stufenweise um $0,5 \mathrm{~mm}$. Die Aufbohrung erfolgt $1 \mathrm{~mm}$ über dem Durchmesser des vorgesehenen Marknagels.

\section{Marknagelung}

Als Implantat eignet sich prinzipiell fast jeder antegrade Tibiamarknagel. Wir haben jedoch im Rahmen einer klinischen Pilotstudie einen speziellen retrograden Tibiamarknagel (RTN, [6]) entwickelt, der sich durch folgende konstruktive Besonderheiten auszeichnet:

- schlanker Nagelschaft von nur $9 \mathrm{~mm}$ Durchmesser,

- konstruktionsbedingte Flexibilität der Marknagelspitze in einer Ebene,

- multiple proximale Verriegelungsmöglichkeit in 3 verschiedenen Ebenen,

- abgeflachte Herzog-Krümmung von nur $15^{\circ}$.

Dieser aus Reintitan gefertigte Marknagel wurde in einer klinischen Erstanwenderstudie an den Berufsgenossenschaftlichen Unfallkliniken Murnau und Frankfurt eingesetzt [6].

In den meisten Fällen lässt sich der Nagel mit der bloßen Hand vorschieben. Wenn nötig, helfen leichte Hammer-

Abb. $1 \nabla$ Hohe Tibiaschaftfraktur mit ausgeprägter Haut- und Weichgewebskontusion am Eintrittspunkt für die antegrade Marknagelung

Abb. $2 \nabla$ Retrograder Tibianagel mit 4facher proximaler und distaler statischer Verriegelung

Abb. $3 \nabla$ Ausheilungszustand 1 Jahr nach Trauma bei bereits erfolgter Materialentfernung
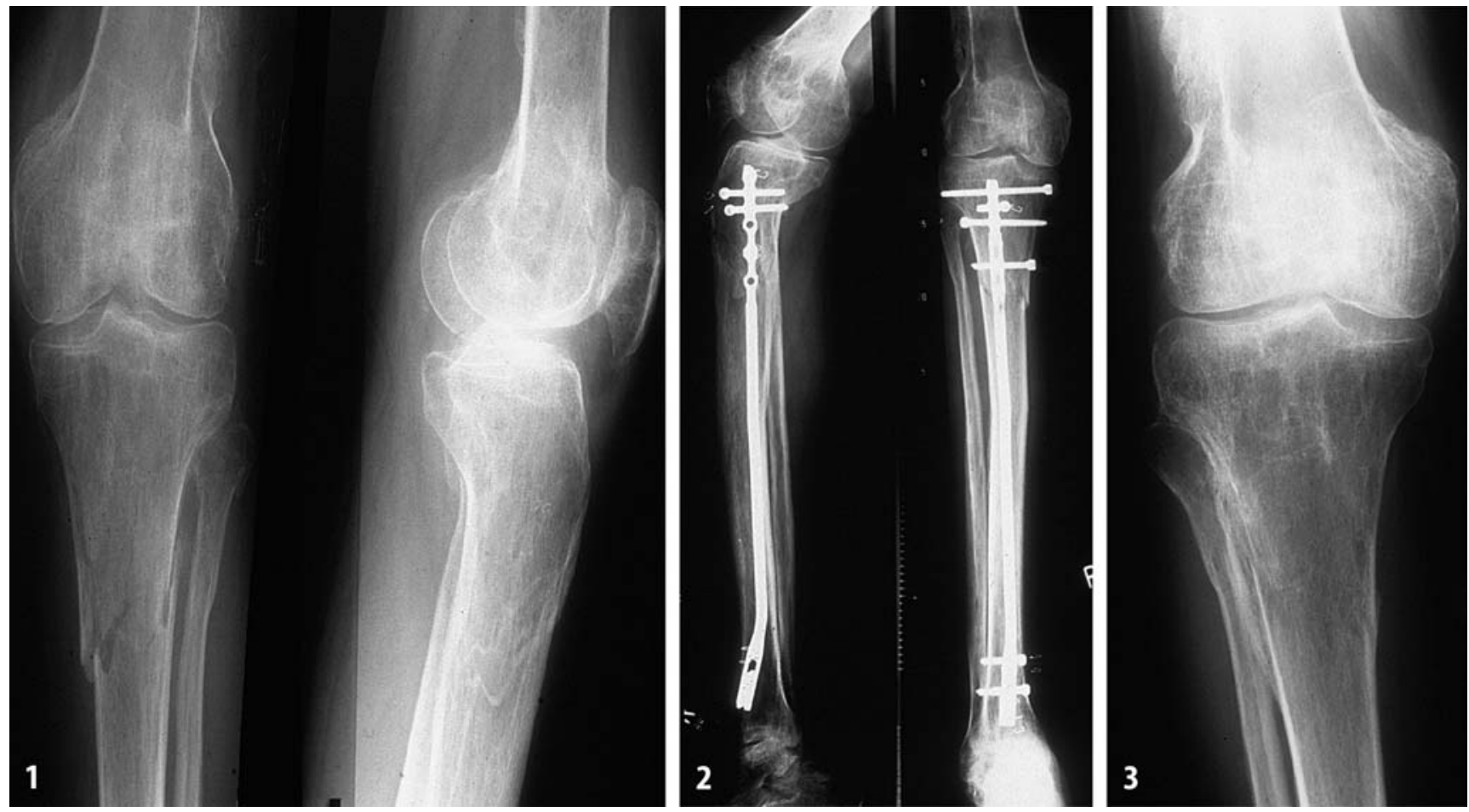

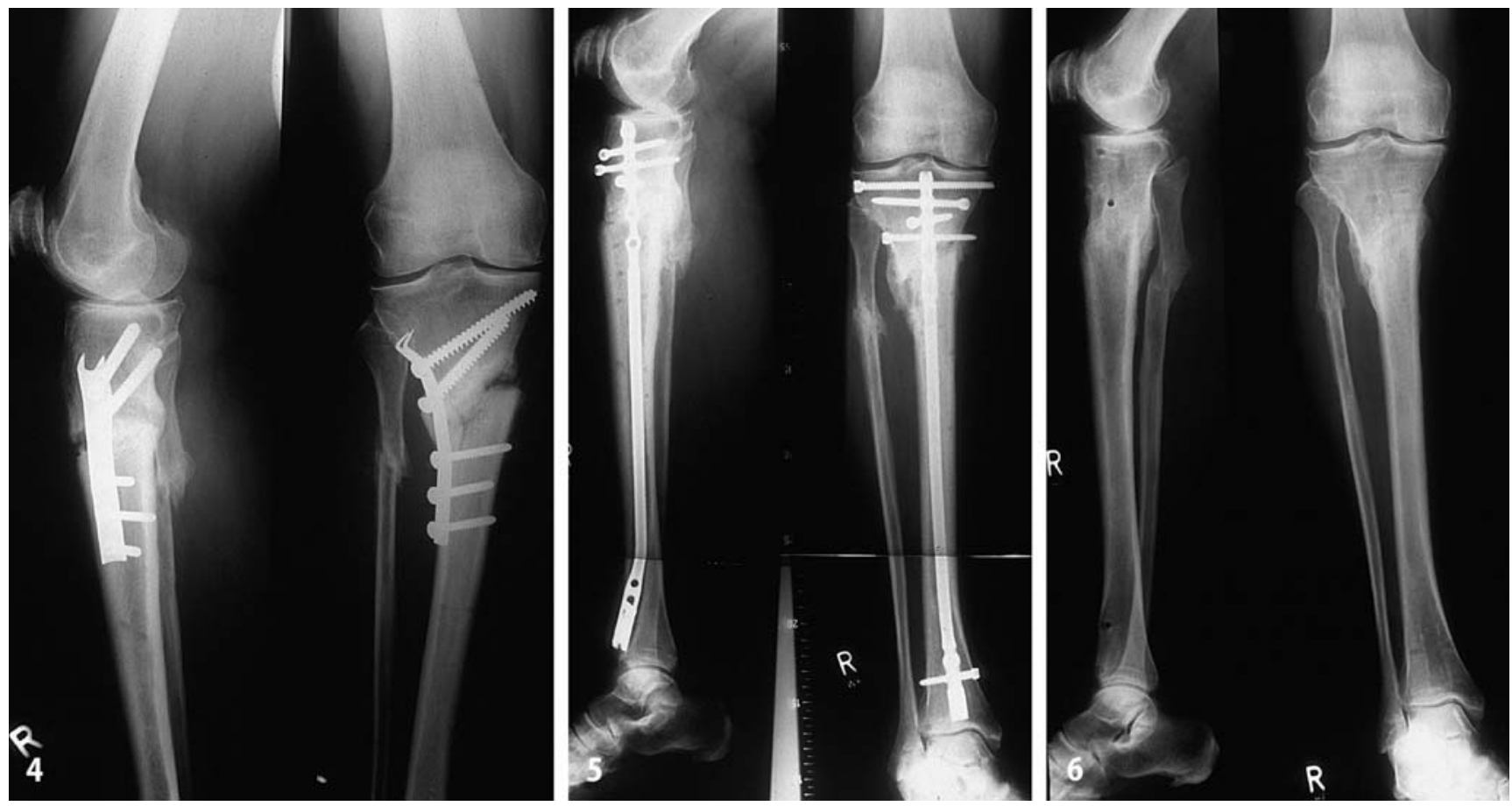

Abb. $4 \Delta$ Zustand nach valgisierender Umstellungsosteotomie und Ausbildung einer Pseudarthrose im proximalen Tibiafünftel

Abb. 5 Korrekturosteotomie mit retrograder Marknagelung, proximal 4fach, distal dynamischkomprimiert verriegelt

Abb. $6 \Delta$ Ausheilungsbild 11 Monate nach Korrektur bei bereits erfolgter Materialentfernung

schläge beim Vortreiben des Marknagels in retrograder Richtung. Auf keinen Fall sollte der Nagel mit Gewalt gegen einen Widerstand vorgetrieben werden. Statt dessen empfiehlt es sich, einen Nagel kleineren Durchmessers zu verwenden. Der Nagel sollte etwas in die Tiefe des Markraumes versenkt werden, um nicht über die Kortikalis der distalen ventralen Tibia vorzustehen. Dabei muss berücksichtigt werden, dass bei Anwendung einer optional vorgesehenen Kompressionsvorrichtung der Marknagel ohnehin wieder etwas nach distal herausgleitet.

\section{Verriegelung}

Die proximale Verriegelung der Verriegelungslöcher an der Marknagelspitze in Höhe des Tibiakopfes erfolgt in Freihandtechnik von verschiedenen Seiten. Dazu wird ein Standard-3,2-mm-Bohrer verwendet. Ein Vorschneiden des Gewindes ist nicht notwendig. Normalerweise werden die Verriegelungsbolzen von medial nach lateral und von ventral nach dorsal eingesetzt. Die distalen Verriegelungslöcher an der Basis des Mark- nagels (statisch und dynamisch) können über das Einführzielgerät verriegelt werden.

\section{Fallbeispiele}

\section{Fallbeispiel: Frakturen im proximalen Tibiafünftel}

- Hohe Tibiaschaftfraktur (Abb. 1) mit ausgeprägter Haut- und Weichgewebskontusion am Eintrittspunkt für die antegrade Marknagelung.

- Postoperatives Versorgungsbild (Abb. 2): retrograder Tibianagel mit 4 facher proximaler und distaler statischer Verriegelung.

- Ausheilungszustand 1 Jahr nach Trauma bei bereits erfolgter Materialentfernung (Abb. 3)

\section{Fallbeispiel: Korrekturosteotomie im proximalen Tibiafünftel}

- Zustand nach valgisierender Umstellungsosteotomie und Ausbildung einer Pseudarthrose (Abb. 4).
- Korrektur mit RTN, proximal 4 fach, distal dynamisch-komprimiert verriegelt (Abb. 5).

- Ausheilungsbild 11 Monate nach Korrektur bei bereits erfolgter Materialentfernung (Abb. 6).

\section{Fallbeispiel: Segmenttransport im proximalen Tibiadrittel zur knöchernen Defektrekonstruktion}

- Autologer Knochenersatz durch Kallusdistraktion und Segmenttransport (Abb. 7).

- Abschluss mit Segmenttransport und Andockmanöver (Abb. 8).

\section{Fallbeispiel: Unterschenkelfraktur bei vorbestehender Kniegelenks- arthrodese}

- Stabilisation mit transartikulärem RTN (Abb. 9).

\section{Ergebnisse}

Bislang wurden 28 retrograde Tibiamarknagelimplantationen vorgenom- 

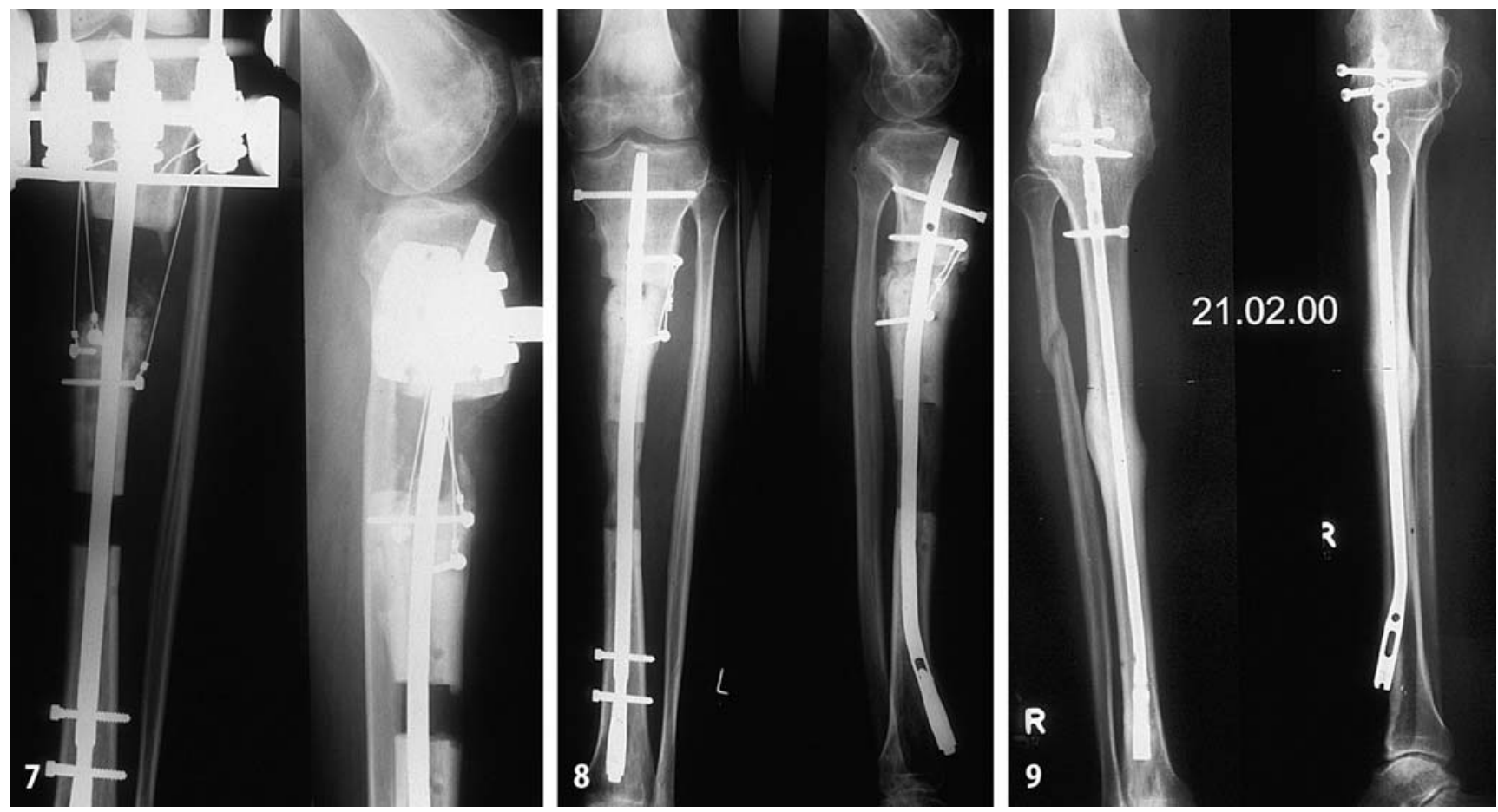

Abb. $7 \Delta$ Autologer Knochenersatz durch Kallusdistraktion und Segmenttransport. RTN: Stabilisationssystem, F.E. + Seilzug: Transportsystem

Abb. $8 \Delta$ Abschluss mit Segmenttransport und Andockmanöver

Abb. $9 \Delta$ Stabilisation einer Unterschenkelfraktur bei vorbestehender Kniegelenksarthrodesemit mit transartikulärem RTN

men: 24 an der Berufsgenossenschaftlichen Unfallklinik Murnau und 4 an der Berufsgenossenschaftlichen Unfallklinik in Frankfurt. Unter Anwendung des in den anatomischen Voruntersuchungen ermittelten idealen Eintrittspunkts konnte der Markkanal in retrogader Richtung mit den flexiblen Bohrwellen über einen eingebrachten Führungsspieß ohne Perforation der ventralen oder dorsalen Kortikalis der Tibia präpariert werden. Unter den ersten $10 \mathrm{Im}$ plantationen kam es bei einem Patienten beim Vortreiben des Marknagels zu einer distalen Tibiafraktur am Marknageleintrittspunkt, die mittels einer zusätzlichen Plattenosteosynthese stabilisiert werden musste. Dagegen verliefen alle anderen Implantationen völlig problemlos.

Nervenirritationen im Bereich des Sprunggelenkes wurden nicht festgestellt. Die Knochenbruchheilung bzw. die knöcherne Konsolidierung der Osteotomien fanden zeitgerecht statt, sodass mittlerweile über 20 der Marknägel wieder entfernt worden sind. Der mittlere Nachuntersuchungszeitraum beträgt der- zeit 30 Monate (6-39 Monate). In keinem Fall kam es zu einer verzögerten Knochenbruchheilung oder Ausbildung einer Pseudarthrose.

Axiale oder rotatorische Korrekturverluste des proximalen Tibiaanteils traten nicht in Erscheinung. Zusätzliche operative Eingriffe (z. B. Sponogiosaanlagerungen) waren nicht erforderlich. In keinem Fall kam es zu einem mechanischen Versagen des Marknagels oder der Verriegelungsschrauben. Ebenso wenig fanden sich radiologische Zeichen einer vorzeitigen Lockerung der Implantatkomponenten.

\section{Diskussion}

Das Konzept der retrogaden Tibiamarknagelung bietet in vielen Fällen von sehr hohen Tibiafrakturen eine vorteilhafte Alternative zur Plattenosteosynthese. Ursprünglich war das Implantat zur Stabilisierung der tibialen Osteotomie bei vaskularisierten allogenen Kniegelenkstransplantationen entwickelt worden. Es kann jedoch in gleicher Weise zur Stabilisierung von Frakturen, Osteotomien, sowie zur Kallusdistraktion bei ganz hohen Tibiadefekten nach septisch bedingtem Knochensegmentverlust eingesetzt werden.

Unsere bisherigen Ergebnisse zeigen, dass der proximale Tibiaanteil durch das Implantat ausreichend stabil fixiert wird. Die geschlossene Repositionstechnik und die abseits der Fraktur liegende Insertionsstelle erweisen sich unter vielen klinischen Bedingungen als vorteilhaft. Besonders bei sehr hohen Tibiafrakturen mit kontusionierten Haut- bzw. Weichteilverhältnissen ist eine Plattenosteosynthese nicht ungefährlich. Eine antegrade, konventionelle Tibiamarknagelung kann in diesen Fällen oftmals nicht zur Anwendung kommen. Die retrograde Tibiamarknagelung bietet für die Versorgung solcher Verletzungen eine Alternative.

\section{„Die retrograde Tibiamarknage- lung bietet für die Versorgung sehr hoher Tibiafrakturen eine Alternative."}


Abschließend soll jedoch klar hervorgehoben werden, dass die anterograde Marknageltechnik an der Tibia sicherlich auch weiterhin das Standardvorgehen bleiben wird und der retrograde Tibiamarknagel für spezielle Ausnahmeindikationen zum Einsatz kommen wird.

\section{Literatur}

1. Angliss RD, Tran TA, Edwards ER, Doig SG (1996) Unreamed nailing of tibial shaft fractures in multiply injured patients. Injury 27: 255-260

2. Danziger MB, Caucci D, Zecher SB, Segal D, Covall DJ (1995) Treatment of intercondylar and supracondylar distal femur fractures using the GSH supracondylar nail. Am J Orthop 24: 684-690

3. Watson T (1994) Current concepts review: treatment of unstable fractures of the shaft of the tibia.J Bone Joint Surg Am 76: 1575-1584

4. Küntscher G, Maatz R (1945) Technik der Marknagelung. Georg Thieme, Leipzig

5. Hofmann GO, Gonschorek O, Bühren V (1999) Retrograde tibial nailing: anatomical considerations and first clinical experiences. Osteosynthese Int 7: 162-169
6. Hofmann GO, Gonschorek O, Bühler M, Bühren V (2001) Retrograde Nagelung der Tibia. Trauma Berufskrankh 3 (Suppl): 303-308

7. Hofmann GO, Kirschner MH, Wagner FD, Brauns L, Gonschorek O, Bühren V (1998) Allogeneic vascularized transplantation of human femoral diaphyses and total knee joints - first clinical experiences. Transplant Proc 30: 2754-2761

8. Hofmann GO, Kirschner MH (2000) Clinical experience in allogenic vascularized bone and joint allografting. Microsurgery 20: 375-383

\title{
H.J. Oestern
}

Klinik für Unfall- und Wiederherstellungschirurgie, Allgemeines Krankenhaus Celle

\section{Diaphysåre Unterschenkelfrakturen. Algorithmus des operativen Vorgehens und differenzierte Indikationsstellung hinsichtlich der Retentionsverfahren}

\author{
Autor hat kein Manuskript eingereicht
}

